\title{
ELABORAÇÃO E ANÁLISE SENSORIAL DE PATÊ DE FRANGO COM OOMEGA-3 E ANTIOXIDANTES NATURAIS DO BAGAÇO DE UVA
}

\section{ELABORATION AND SENSORIAL ANAL YSIS OF CHICKEN PATE WITH OMEGA-3 AND NATURAL ANTIOXIDANTS FROM GRAPE POMACE}

\author{
Karina Suseth Rodrigues Da Silva Cechin ${ }^{{ }^{\star}}$, Daniela Miotto Bernardi ${ }^{2}$ \\ ${ }^{1}$ Acadêmica de Nutrição, Centro Universitário Fundação Assis Gurgacz (FAG). ${ }^{2}$ Nutricionista \\ Doutora, Docente do Curso de Nutrição, Centro Universitário Fundação Assis Gurgacz (FAG). \\ * Autor correspondente: karinasusethrd@gmail.com - https://orcid.org/0000-0003-0168-3993
}

DOI: $10.35984 / f j h . v 2 i 1.139$

\section{RESUMO}

O bagaço de uva é um subproduto da indústria vinícola, que vem ganhando grande interesse pela sua capacidade antioxidante. O óleo de canola é fonte de vitamina $E$ e ômega 3 . $O$ objetivo desse estudo foi a análise do efeito antioxidante do bagaço de uva em patês de frango elaborados com adição de ômega-3, assim como a verificação da aceitação do produto por meio de uma análise sensorial. Foram realizadas quatro formulações de patês de frango e posteriormente feita análise sensorial de pequenas amostras. Os materiais utilizados para cada $100 \mathrm{~g}$ de produto foram aproximadamente: $33 \%$ de filé "sassami" de frango, $17 \%$ de cebola, $12 \%$ de tomate, $5 \%$ de bagaço de uva, $26 \%$ de óleo de canola, $25 \%$ de óleo de milho, $8 \%$ de leite. Também foram utilizados os seguintes temperos: $1 \%$ de salsinha e $0,30 \%$ de sal. $\mathrm{Na}$ análise sensorial participaram 120 pessoas que avaliaram atributos do produto, sendo eles: aparência, aroma, textura, sabor e intenção de compra, tanto de um patê existente no mercado como do patê desenvolvido para este trabalho. Em conclusão as amostras B e BC, que possuíam bagaço de uva na sua formulação não foram bem aceitas pelos provadores, no entanto as amostras C, P e Comercial, que não tinham bagaço de uva, foram aprovadas. Também foi observado que a inclusão do óleo de canola não interferiu na nota, portanto, foi possível produzir um patê de frango com maior teor de a-linolênico sem prejuízo sensorial e alta intenção de compra.

Palavras chaves: Subproduto, Patê, Funcional, Produção.

\begin{abstract}
Grape pomace is a by-product of the wine industry, which has been gaining great interest for its antioxidant capacity. Canola oil is a source of vitamin $E$ and omega 3. The aim of this study was to analyze the antioxidant effect of grape pomace in chicken pate with added omega-3 on its recipe, as well as verification of product acceptance through sensory analysis. Four chicken pate formulations were made and then sensory analysis of small samples. The materials used for each $100 \mathrm{~g}$ of product were approximately: 33\% chicken sassami fillet, $17 \%$ onion, $12 \%$ tomato, $5 \%$ grape pomace, $26 \%$ canola oil, $25 \%$ corn oil, $8 \%$ milk. The following seasonings were also used: $1 \%$ parsley and $0.30 \%$ salt. In the sensory analysis participated 120 people who evaluated product attributes, which were: appearance, aroma, texture, taste and purchase intention, both of a pate existing in the market and the pate developed for this work. In conclusion, samples $B$ and $B C$, which had grape marc in their formulation, were not well accepted by the tasters, however samples C, $P$ and Comercial, which did not have grape pomace, were approved. It was also observed that the inclusion of canola oil did not interfere in the grade, therefore, it was possible to produce a chicken pate with higher $\alpha$-linolenic content without sensory impairment and high purchase intention.
\end{abstract}

Keywords: Subproduct, pate, Functional, Production. 


\section{INTRODUÇÃO}

Atualmente, por questões sustentáveis, tem sido mais observado e estudado o aproveitamento industrial dos subprodutos do processamento de alimentos, e no que se refere a indústria vinícola, alguns dados estimam que para produzir 100 litros de vinho branco é obtido aproximadamente $31,17 \mathrm{~kg}$ de subprodutos, dos quais aproximadamente $20 \mathrm{~kg}$ são de bagaço de uva, e $25 \mathrm{~kg}$ de subprodutos para o mesmo volume de vinho tinto (CAMPOS, 2005; COSTA E BELCHIOR,1972).

As uvas são consideradas uma das maiores fontes de compostos fenólicos quando comparadas a outras frutas. A capacidade funcional das uvas tem sido analisada mais detalhadamente nesses últimos anos, devido a estudos que tem mostrado múltiplos efeitos biológicos, positivamente relacionados aos compostos fenólicos das mesmas, tais como a atividade antioxidante, anti-inflamatória, antimicrobiana e anticarcinogênica. Dentre todos os compostos fenólicos das uvas, o resveratrol tem atraído maior atenção, por ter mostrado relação com a redução de risco de doenças cardiovasculares. Alguns estudos também têm afirmado que quanto mais intensa a coloração da uva, maior é o conteúdo dos compostos fenólicos e, portanto, apresenta maior capacidade antioxidante (ABE et al., 2007).

Os principais compostos fenólicos encontrados na uva são os flavonoides (antocianinas e flavonóis), estilbenos (resveratrol), ácidos fenólicos (derivados dos ácidos cinâmicos e benzoicos) e uma grande variedade de taninos. As antocianinas

são efetivas doadoras de hidrogênio e seu potencial antioxidante depende do número e da posição dos grupos hidrogênios e suas conjugações, além da presença de elétrons nos anéis benzênicos (MALACRIDA, 2005; ROCKENBACH, 2008).

Os lipídeos representam um papel importante quando se trata da qualidade e características físicas do alimento, como aroma, sabor, textura e aparência, além de aportar valor nutritivo por se tratar de um macronutriente, constituindo uma fonte de energia metabólica, vitaminas lipossolúveis e ácidos graxos essenciais como os presentes na canola (LEISTENSCHNEIDER, 2015; ST. ANGELO et al., 1996).

O óleo de canola é um exemplo de alimento vegetal rico em ômega-3, cujo nome é derivado de Canadian Oil Low Acid que quer dizer "óleo canadense de pouca acidez", e sua principal característica é a sua composição de ácidos graxos, pois além do ômega-3, este apresenta baixo teor de gordura saturada, alto teor de vitamina $\mathrm{E} \mathrm{e}$ gorduras monoinsaturadas, sendo que cada $100 \mathrm{~g}$ de óleo de canola apresenta aproximadamente $6,8 \mathrm{~g}$ de ácido graxo a-linolênico (ANGELOTTI-MENDONÇA et al., 2016; NEPA - UNICAMP, 2011).

Além disso, os benefícios do consumo do ômega-3 vão desde a gestação, redução de risco de doenças cardiovasculares, desenvolvimento visual e cognitivo na infância, prevenção de doenças neurodegenerativas, redução de mecanismos proinflamatórios, devido a melhorar a coagulação sanguínea e na influência da trombose, na pressão sanguínea e nos mecanismos inflamatórios, podendo dessa forma ajudar na limpeza dos vasos sanguíneos e também favorecendo a diminuição da presença de partículas de colesterol LDL que quando acumuladas, são prejudiciais para o sistema circulatório e no geral, na saúde das pessoas (RAMOS; RAMOS, 2005; SANTOS; BORTOLOZO, 2008).

Apesar dos efeitos benéficos, a incorporação de ômega-3 em alimentos resulta no aumento de chance de oxidação lipídica, o qual é um processo natural inevitável que implica diretamente com o valor comercial dos alimentos, por se tratar da principal 
causa da deterioração desses ácidos graxos, causando como consequência a mudança negativa nas características organolépticas do produto, como a rancificação, pois representa para o consumidor uma importante causa de depreciação ou rejeição (LEISTENSCHNEIDER, 2015; ST. ANGELO et al., 1996).

A oxidação lipídica ocorre quando o oxigênio entra em contato com as espécies reativas de oxigênio (ROS), também conhecidos como radicais livres, que são definidos como espécies químicas com um ou mais elétrons desemparelhados, altamente reativos e capazes de atacar qualquer biomolécula, e de meia vida curta. Sua formação ocorre durante os processos oxidativos biológicos, sendo assim formados fisiologicamente nos sistemas biológicos a partir de compostos endógenos (RENZ, 2002).

Os antioxidantes em alimentos são compostos que podem retardar ou evitar as reações de oxidação provocadas pelos radicais livres. A atuação deles ocorre pela doação de hidrogênio e/ou transferência de elétrons para o radical livre, sendo chamados sequestradores de radicais livres, inibindo sua forma reativa. Devido a isto, é interessante estudar a aceitação da adição de antioxidantes em produtos inovadores, de forma a poder oferecer maior vida de prateleira e maior funcionalidade ao alimento (BOROSKI et al.,2015).

Diante do exposto, o objetivo do estudo foi analisar o efeito antioxidante do bagaço de uva em patês de frango elaborados com adição de ômega-3, assim como a verificação da aceitação do produto por meio de uma análise sensorial.

\section{METODOLOGIA}

\subsection{Matéria prima}

Este estudo foi realizado no laboratório de nutrição de um Centro Universitário localizado na cidade de Cascavel, no estado Paraná, onde foi elaborado o patê com alimentos naturais. Para elaboração do patê foram utilizados: o bagaço da uva do tipo bordô, adquirido na vinícola DEZAN, localizada em Três Barras do Paraná, como doação para a realização do estudo. Os demais ingredientes: filé de frango "sassami", óleo de girassol, óleo de canola, leite, tomate, cebola, sal e salsinha foram adquiridos em um supermercado local da cidade de Cascavel - PR.

\subsection{Processamento do bagaço de uva}

Após cinco dias de fermentação, o bagaço da uva foi coletado e pesado. Foi levado para uma estufa de esterilização e secagem da marca Ethick, em uma temperatura contínua de $55^{\circ} \mathrm{C}$ por um prazo de 96 horas, até estar totalmente seco, ao término deste processo foi pesado novamente. Posteriormente foi passado por um liquidificador industrial até obter a consistência de farinha. Foram verificados aspectos relacionados à rendimento de secagem.

\subsection{Formulação e elaboração do produto}

Para aplicação comercial do bagaço de uva desidratado, foi escolhido formulação de patê com maior teor de ômega-3, sendo utilizado a formulação comercial como padrão para comparação.

Além da formulação comercial, que foi utilizada como base para a escolha dos ingredientes do novo patê, foram realizadas quatro formulações do produto, sendo classificadas como: Formulação comercial; Formulação P (padrão - sem óleo de canola e sem bagaço da uva); Formulação B (com bagaço da uva); Formulação C 
(com óleo de canola); Formulação BC (com óleo de canola e com bagaço da uva). Na tabela 01 estão apresentadas as informações referentes à formulação empregada nos patês.

Tabela 01. Ingredientes (em percentual) empregados na formulação de patês de frango com e sem incorporação de ômega-3 e antioxidantes naturais.

\begin{tabular}{lcccc}
\hline Ingredientes & Formulação C & Formulação BC & Formulação P & Formulação B \\
\hline Filé "Sassami” Cozido & 33,22 & 31,63 & 33,22 & 31,63 \\
Tomate & 17,28 & 16,45 & 17,28 & 16,45 \\
Cebola & 12,40 & 11,81 & 12,40 & 11,81 \\
Leite & 8,86 & 8,44 & 8,86 & 8,44 \\
Óleo de Canola & 26,58 & 25,31 & 0 & 0 \\
Óleo de Milho & 0 & 0 & 26,58 & 25,31 \\
Sal & 0,33 & 0,32 & 0,33 & 0,32 \\
Salsinha & 1,33 & 1,27 & 1,33 & 1,27 \\
Bagaço de Uva & 0,00 & 4,78 & 0,00 & 4,78 \\
\hline
\end{tabular}

O filé de frango foi refogado em uma panela antiaderente, desfiado e reservado. A cebola e o tomate foram cortados em pedaços pequenos e fritos na mesma panela do frango para aproveitar a gordura e o caldo da carne. Para fazer a maionese de leite com óleo de canola, foi colocado o leite no liquidificador junto com o sal e batido constantemente, adicionando aos poucos o óleo de canola até chegar no ponto de espessura desejado. Para a maionese de leite com óleo de milho foi repetido o procedimento. A receita de frango com tomate e cebola foi dividida em duas partes iguais, pois uma metade teve a receita de maionese de óleo de canola adicionada e a outra metade a maionese de óleo de milho. Uma vez que estes ingredientes ficaram prontos foi misturado tudo em um processador, junto com o bagaço de uva e processado até alcançar a consistência desejada do patê. Na figura 01, estão apresentados os processos empregados na formulação do produto.

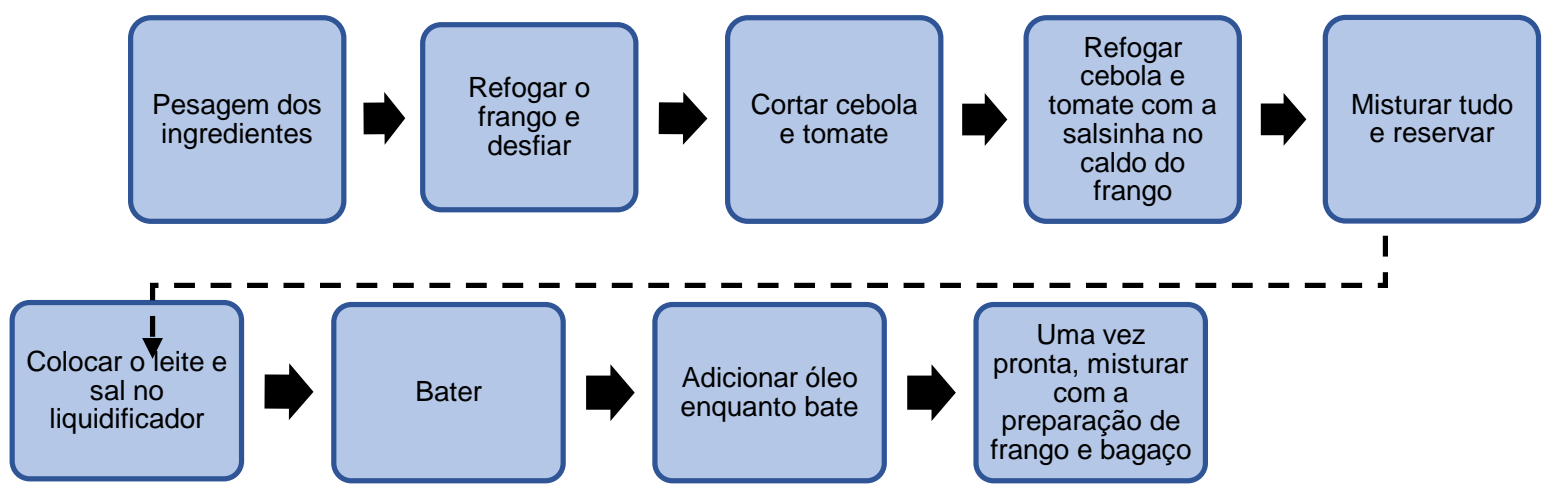

Figura 1. Fluxograma do preparo do patê.

\subsection{Avaliação nutricional do patê}

Tendo como referência a Tabela Brasileira de Composição de Alimentos (TACO) e a tabela de Valores Nutricionais dos alimentos do United States Department of Agriculture (USDA) foi previamente realizado o cálculo da porcentagem dos ingredientes utilizados para a formulação do patê para assim obter o valor nutricional. 
Para os ingredientes filé de "Sassami" cozido, tomate, cebola, leite, óleo de canola, óleo de milho, sal e salsinha foram tomados os valores de energia kcal, proteínas, lipídeos, carboidratos, colesterol e ácidos graxos 18:2 n-6 e 18:3 n-3 das tabelas supracitadas. Para o bagaço de uva a referência utilizada para o cálculo nutricional foi o artigo de Oliveira et al., (2016).

\subsection{Análise sensorial}

Após a aprovação do projeto pelo Comitê de Ética e Pesquisa com seres Humanos, parecer $\mathrm{n}^{0}$ 2.617.581, foi realizada a análise sensorial em um Centro Universitário da cidade de Cascavel-PR, com um grupo de 120 pessoas voluntárias, de ambos sexos e com faixa etária entre os 18 e 40 anos, que leram e concordaram com o Termo de Consentimento Livre e Esclarecido.

Cada participante recebeu uma amostra de um patê comercial e quatro amostras do patê formulado, que correspondem as formulações $P, B, C$ e BC, em copos descartáveis, sem especificação do conteúdo para não influenciar na opinião dos voluntários, que posteriormente fizeram a avaliação preenchendo uma ficha de análise, utilizando uma escala hedônica estruturada de nove pontos em que os extremos de notas variaram entre "Gostei muito (9)" a "Desgostei muitíssimo (1)". A avaliação da intenção de compra também contou com uma escala estruturada de cinco pontos, onde as notas variaram entre dois extremos: "Certamente compraria (5)" e "Certamente não compraria (1)". As informações coletadas foram analisadas estatisticamente para a obtenção dos resultados (DUTCOSKI, 2013) (APENDICE II).

A análise sensorial ocorreu nos dias 08 e 09 de abril de 2019 e foram servidas as cinco amostras de forma aleatória e sem repetição (MACFIE et al., 1989), entregues com copos codificados com algarismos de três dígitos e o peso foi padronizado de $3 \mathrm{~g}$ para cada copo, acompanhado de um copo de água por participante para a limpeza do palato.

Os dados coletados durante a análise sensorial foram posteriormente tabulados no programa Microsoft Office Excel, nos quais foram submetidos a análise de variância (ANOVA) e ao teste de Tukey, para a obtenção dos resultados.

\section{RESULTADOS E DISCUSSÃO}

\subsection{Rendimento do bagaço de uva}

O bagaço de uva foi pesado depois da fermentação ainda úmido, totalizando $14,729 \mathrm{~kg}$. Após a secagem, foi pesado novamente e o valor total do peso do produto foi de $4,267 \mathrm{~kg}$, representando a perda de aproximadamente $71 \%$ do seu conteúdo de água. Uma vez processado no liquidificador industrial, para conseguir o aspecto de farinha foi pesado novamente, totalizando $4,248 \mathrm{~kg}$ de produto obtidos para a realização deste estudo.

Embora a produção da farinha de bagaço de uva tenha apresentado um baixo rendimento, de apenas $30 \%$ do produto, pode-se dizer que é válida a sua utilização, pois o aproveitamento de cascas, galhos, engaços e outros componentes, normalmente descartados pela indústria de alimentos, é uma alternativa sustentável para diminuir o impacto ambiental, promover o combate da fome, alcançar o aproveitamento total dos alimentos, promover a segurança alimentar, além de acrescentar valor nutricional aos alimentos e promover o consumo consciente (DAMIANI et al., 2011). 


\subsection{Análise sensorial e intenção de compra}

Como resultado da análise tivemos que as amostras apresentaram uma grande diferença entre elas, na tabela 2 a seguir, observamos que existe uma notável diferença entre a média das amostras C, P e Comercial, para aceitação global, aparência, aroma, sabor e textura, referente aos seus respectivos desvios padrão, apresentando uma média relativamente alta. As amostras B e BC apresentaram uma média mais baixa, em comparação com as amostras mencionadas anteriormente, referente aos seus respectivos desvios padrão. Isto demonstra que as amostras que tiveram a inclusão do bagaço de uva (amostra B e BC) não foram tão bem aceitas, como as que não tiveram bagaço de uva incluso na receita (amostras $\mathrm{C}, \mathrm{P}$ e Comercial). Assim também é possível observar que a inclusão do óleo de canola, como fonte de ômega-3 e ômega-6 não alterou significativamente as características sensoriais do produto. Características estas demonstradas nos resultados da amostra C que teve uma ótima aceitabilidade e na amostra 4 que a inclusão do mesmo não interferiu nem positivamente nem negativamente na nota dada pelos provadores, assume-se que o mesmo pode passar quase desapercebido.

Tabela 2. Resultados da análise sensorial de patês de frango com incorporação de ômega-3 e bagaço de uva, os dados estão apresentados em média e desvio padrão.

\begin{tabular}{lcccccc}
\hline ATRIBUTOS & $\begin{array}{c}\text { Formulação } \\
\text { C }\end{array}$ & $\begin{array}{c}\text { Formulação } \\
\text { B }\end{array}$ & $\begin{array}{c}\text { Formulação } \\
\text { P }\end{array}$ & $\begin{array}{c}\text { Formulação } \\
\text { BC }\end{array}$ & $\begin{array}{c}\text { Formulação } \\
\text { Comercial }\end{array}$ & $\begin{array}{c}\text { VALOR DE } \\
\text { P }\end{array}$ \\
\hline $\begin{array}{l}\text { ACEITAÇÃO } \\
\text { GLOBAL }\end{array}$ & $7,1 \pm 1,7 \mathrm{~A}$ & $4,8 \pm 2,5 \mathrm{C}$ & $7,5 \pm 1,5 \mathrm{~A}$ & $4,7 \pm 2,4 \mathrm{C}$ & $6,2 \pm 2,2 \mathrm{~B}$ & $<0,001$ \\
& & & & & & \\
APARÊNCIA & $7,2 \pm 1,6 \mathrm{~A}$ & $7,0 \pm 2,4 \mathrm{C}$ & $5,6 \pm 1,8 \mathrm{~A}$ & $4,3 \pm 2,3 \mathrm{C}$ & $4,2 \pm 2,2 \mathrm{~B}$ & $<0,001$ \\
AROMA & $6,9 \pm 1,7 \mathrm{~A}$ & $4,7 \pm 2,3 \mathrm{C}$ & $7,0 \pm 1,9 \mathrm{~A}$ & $4,6 \pm 2,4 \mathrm{C}$ & $6,2 \pm 2,1 \mathrm{~B}$ & $<0,001$ \\
SABOR & $6,7 \pm 2,0 \mathrm{C}$ & $4,4 \pm 2,7 \mathrm{~B}$ & $7,6 \pm 1,7 \mathrm{~A}$ & $4,4 \pm 2,6 \mathrm{~B}$ & $6,1 \pm 2,4 \mathrm{~A}$ & $<0,001$ \\
TEXTURA & $7,0 \pm 1,7 \mathrm{~B}$ & $4,6 \pm 2,5 \mathrm{C}$ & $7,1 \pm 1,7 \mathrm{~A}$ & $4,6 \pm 2,6 \mathrm{~B}$ & $6,1 \pm 2,1 \mathrm{C}$ & $<0,001$ \\
\hline
\end{tabular}

$A, B, C$ - Letras diferentes na mesma linha mostram diferença significativa no teste de Tukey.

Referente a intenção de compra, representada na Figura 2 a seguir, pode-se observar que as amostras $\mathrm{C}$ e $\mathrm{P}$ tiveram as maiores notas, sendo em sua maioria notas entre " 4 " e " 5 ", que na escala significaria que a maioria dos provadores "certamente comprariam" o produto. A amostra comercial permaneceu com notas mais neutras, em sua maioria sendo avaliada com nota " 3 " que representaria a dúvida na possibilidade da compra para consumo desse produto, e finalizando com as amostras B e BC que apresentaram as menores notas de intenção de compra, tendo sido avaliadas as duas de forma igual pelos provadores, com a nota mínima de intenção de compra "1", sendo representada na escala como "certamente não compraria".

Devido a inexistência de estudos relacionados a formulação de patê de frango com adição de bagaço de uva e ômega-3, a comparação de resultados tanto da análise sensorial como da intenção de compra do patê foi realizada com base em produtos diferentes que também tiveram adição de bagaço de uva ou semelhantes na formulação. Segundo um estudo realizado por Joel Pimentel de Abreu (2018), no qual foi elaborado um biscoito tipo cookies com adição de farinha da casca de uva orgânica, verificou que é possível obter um produto funcional, com alta aceitabilidade, desenvolvido com esse ingrediente. 
Foram realizadas distintas formulações do biscoito, que tiveram a mesma quantidade de farinha de casca de uva na sua composição, mudando apenas a quantidade de gorduras interesterificadas e a quantidade de sacarose, e realizada posteriormente uma análise sensorial, com uma escala hedônica estruturada de nove pontos, que teve como resultado uma melhor aceitação dos cookies onde havia maior porcentagem de gorduras e sacarose. No estudo é citado Ramos e Stein (2000), que afirmam que os alimentos com maior teor de gordura são mais palatáveis, pois algumas substancias voláteis que dão o respectivo sabor ao alimento são solúveis em gorduras, acrescentando também uma textura cremosa e fofa, que pode se tornar uma característica positiva na hora da avaliação. Isto é considerável para o biscoito tipo cookie e o patê, dada a quantidade de gordura que compõe o produto.

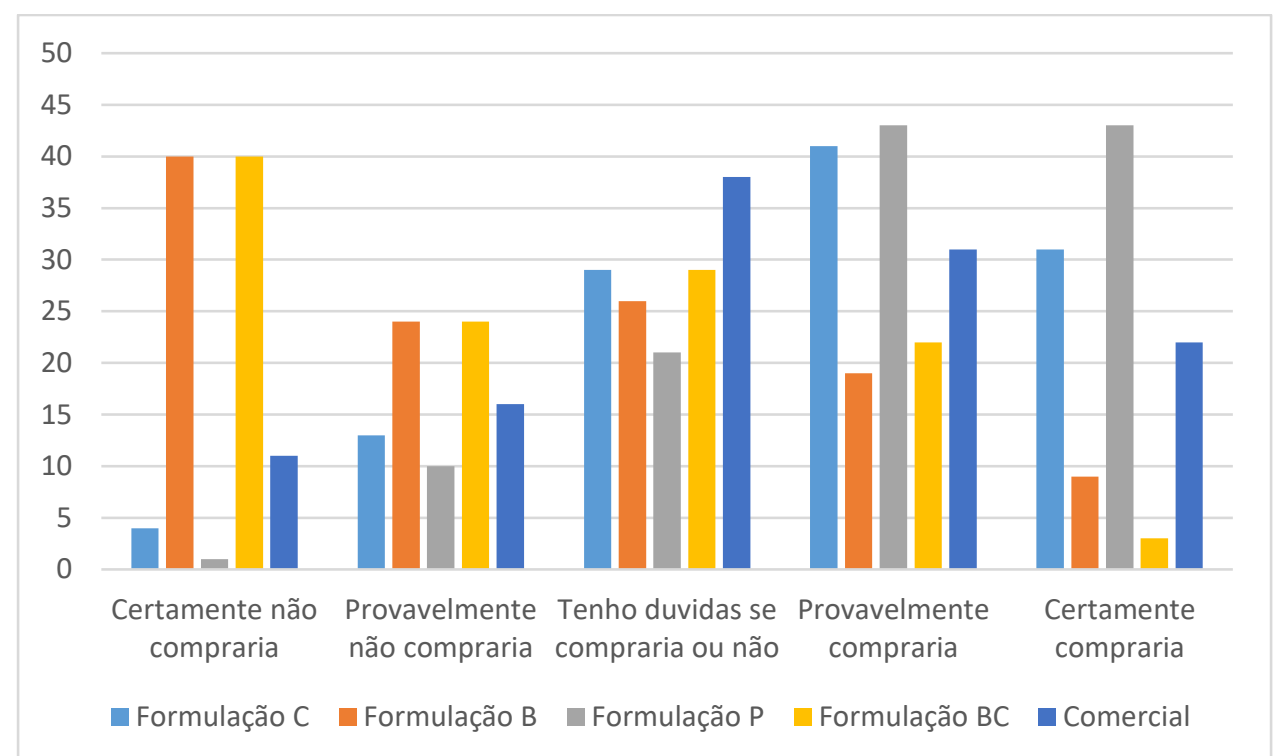

Figura 2. Gráfico de intenção de compra.

Em um estudo de aceitação de um patê de tilápia realizado por Furtado, Resende e Freitas (2010), publicada pela Empresa Brasileira de Pesquisa Agropecuária (EMBRAPA), foi observado que o produto teve uma alta aceitabilidade apresentando médias entre seis e sete na escala hedônica de nove pontos, podendo ser considerado como uma média alta. O patê teve em sua formulação adição de óleo de canola e mesmo não sendo o foco do estudo, pode-se observar novamente como o óleo de canola não interferiu nas características sensoriais do produto, de forma que é possível indicar o uso do mesmo de maneira mais frequente na elaboração de outras receitas. É estimado que quanto mais próximo o consumo do valor diário recomendado dos ômegas, melhores são seus efeitos na diminuição da taxa de mortalidade, de doenças cardiovasculares, de inflamações e outras patologias como artrite reumática e sintomas de asma, ao mesmo tempo que auxilia no fortalecimento do sistema nervoso central (ANTUNES MARTIN et al., 2006).

\subsection{Composição Nutricional dos patês}

Na tabela 3 estão apresentados os resultados da composição nutricional de $100 \mathrm{~g}$ de cada formulação de patê produzido.

Observa-se que os patês $\mathrm{C}$ e $\mathrm{P}$, que não tiveram inclusão do bagaço de uva na sua formulação apresentaram maior valor energético, proteico e lipídico que as 
formulações $B, B C$ e Comercial. Também se observa que as formulações $B$ e $B C$ apresentaram uma diferença de $2 \mathrm{~g}$ de carboidratos a mais do que as formulações $\mathrm{C}$ e P, com a adição do bagaço de uva consequentemente apresentaram um teor de fibras mais elevado, de aproximadamente $0,7 \mathrm{~g}$. Em relação aos valores de 18:3 (ômega-3), observou-se que as formulações C e BC, que possuíam óleo de canola, apresentaram maior quantidade de ômega- 3 que as formulações $B$ e $P$ e que de forma inversa, as formulações B e P apresentaram maior teor de ômega-6. Para o patê comercial não foram obtidos os valores de 18:2 e 18:3, os valores de lipídios e calorias foram significativamente mais baixos que os valores dos patês formulados para 0 estudo, não apresentou fibras nem carboidratos e as proteínas apresentaram uma diferença de apenas $1 \mathrm{~g}$.

Tabela 3. Composição nutricional de $100 \mathrm{~g}$ de patê

\begin{tabular}{lccccc}
\hline Nutriente & FC & FB & FP & FBC & Comercial \\
\hline Kcal & 299,60 & 285,29 & 299,60 & 285,29 & 270 \\
Carboidrato (g) & 2,07 & 4,08 & 2,07 & 4,08 & 0 \\
Proteína (g) & 11,32 & 11,14 & 11,32 & 11,14 & 10 \\
Lipídios (g) & 27,63 & 26,63 & 27,63 & 26,63 & 24 \\
Fibras (g) & 0,48 & 1,18 & 0,48 & 1,18 & 0 \\
C18:3 (g) & 1,82 & 0,26 & 0,27 & 1,73 & - \\
C18:2 (g) & 5,68 & 12,77 & 13,41 & 5,41 & - \\
\hline
\end{tabular}

${ }^{*}$ Formulação $\mathrm{C}$ - com óleo de canola; Formulação $\mathrm{B}$ - com bagaço de uva; Formulação $\mathrm{P}$ - sem óleo de canola e sem bagaço de uva; Formulação BC- com óleo de canola e bagaço de uva; Formulação Comercial.

\section{CONSIDERAÇÕES FINAIS}

As amostras $B$ e $B C$, que possuíam bagaço de uva na sua formulação não foram bem aceitas pelos provadores quando comparadas às as amostras $\mathrm{C}, \mathrm{P}$ e Comercial que não tinham bagaço de uva, também foi observado que a inclusão do óleo de canola não interferiu na nota. A aceitação sensorial do produto refletiu diretamente na intenção de compra, de forma que as amostras $\mathrm{C}$ e $\mathrm{P}$ tiveram nota mais alta em comparação com as amostras B e BC. Portanto, embora a aceitação da adição de bagaço de uva não tenha sido alta, foi possível produzir um patê de frango com maior teor de a-linolênico (ômega-3) sem prejuízo sensorial. É observada também a necessidade de quantificar a atividade antioxidante em diversos produtos em futuras pesquisas e verificar a aceitabilidade da inclusão do bagaço de uva em diferentes concentrações, para assim promover a sustentabilidade no âmbito industrial além de aproveitar mais amplamente um alimento tão benéfico como a uva.

\section{REFERÊNCIAS}

ABE, L. T. et al. Compostos fenólicos e capacidade antioxidante de cultivares de uvas Vitis labrusca L. e Vitis vinifera L. Ciência e Tecnologia de Alimentos, v. 27, n. 2, p. 394-400, 2007.

ABREU, J. P. de. Efeitos da adição de farinha de casca de uva orgânica (Vitis Labrusca) sobre as características físicas, químicas e sensoriais no desenvolvimento de biscoito tipo cookie com alegação funcional. v. 1 , p. 88 , 2018. 
ANGELOTTI-MENDONÇA, J. et al. Canola. n. August 2018, 2016.

ANTUNES MARTIN, C. et al. Omega-3 and omega- 6 polyunsaturated fatty acids: importance and occurrence in foods Makoto MATSHUSHITA 1. Rev. Nutr, v. 19, n. 6, p. $761-770,2006$.

ARVANITOYANNIS, I. S.; LADAS, D.; MAVROMATIS, A. Potential uses and applications of treated wine waste: A review. International Journal of Food Science and Technology. v. 41, n. 5, p. 475-487, 2006.

BOROSKI, M. et al. Antioxidantes- Princípios e métodos analíticos. Editora Appris Ltda. 1 Edição, 2015.

CAMPOS, L. M. A. S. de. Obtenção de Extratos de Bagaço de uva Cabernet Sauvingnon (Vitis vinifera): Parâmetros de Processo e Modelagem Matemática. p. 141, 2005.

COSTA, J.E.; BELCHIOR, A.P. Laboração e utilização dos produtos secundários da vinificação. Relatório nacional, Lisboa, 1972.

DAMIANI, C. et al. Doces de corte formulados com casca de manga. Pesquisa Agropecuaria Tropical, v. 41, n. 3, p. 360-369, 2011.

DUTCOSKI, S. D. Análise Sensorial de alimentos. 4. ed. Curitiba - PR: Editora Universitária Champagnant, 2013.

FURTADO, A. A. L.; RESENDE, A. L. da S. S.; FREITAS, D. de G. C. Aceitabilidade do Patê de Tilápia. Comunicado Técnico da Embrapa, p. 4, 2010.

LEISTENSCHNEIDER, D. Einfluss von Transportporensytemen auf die elektrochemische Energiespeicherfähigkeit

Kohlenstoffmaterialien. v. 22, n. 1, p. 4-6, 2015.

MACFIE, H. J. et al. Designs to balance the effect of order of presentation and first-order carry-over effects in hall tests. Journal of sensory studies, v. 4, n. 2, p. $129-148,1989$.

MALACRIDA, C. R. Compostos fenólicos totais e antocianinas em suco de uva 1. v. 25, n. 4, p. 659-664, 2005.

NEPA - UNICAMP. Tabela brasileira de composição de alimentos. NEPA Unicamp, p. 161, 2011. Disponível em: http://www.unicamp.br/nepa/taco/. Acesso em 20 mai. 2019.

OLIVEIRA, R. . et al. Composição centesimal de farinha de uva elaborada com bagaço da indústria vitivinícola. v. 1, p. 2-7, 2016. 
RAMOS, S.; RAMOS, M. E. M. Dieta e Risco Cardiovascular: ômega 3, óleo de oliva, oleaginosas,... O que é fato? Revista da Sociedade de Cardiologia do Rio Grande do Sul, v. 6, p. 10-12, 2005.

RAMOS, M.; STEIN, L. M. Desenvolvimento do comportamento alimentar infantil Development of children's eating behavior. Jornal de Pediatria, v. 76, p. 229-237, 2000.

RENZ, S. V. Oxidação e Antioxidantes. Estresse Oxidativo e Antioxidante. p. 1519, 2002.

ROCKENBACH, I. I. Compostos fenólicos, ácidos graxos e capacidade antioxidante do bagaço da vinificação de uvas tintas (Vitis vinifera L. e Vitis labrusca L.). p. 113, 2008.

SANTOS, L. E. S.; BORTOLOZO, E. A. F. Q. Potenciais benefícios no metabolismo lipídico omega 3 intake: considerations on the potential benefits in lipid metabolism. v. 14, n. 2, p. 161-170, 2008.

ST. ANGELO, A. J. et al. Lipid Oxidation in Foods - an Overview. [S.I: s.n.], 1996. USDA. Nutritive Value of Foods Nutritive Value of Foods. Usda, n. 72, p. 103, 2016. 